\title{
TRIP-1--A TWO-DIMENSIONAL P-3 PROGRAM IN X-Y GEOMETRY FOR THE IBM-704
}

July 1960

CONTRACT AT-11-1-GEN-14

BETTIS ATOMIC POWER LABORATORY PITTSBURGH, PENNSYLVANIA

Operated for the U.S. ATOMIC ENERGY COMMISSION bY WESTINGHOUSE ELECTRIC CORPORATION 


\section{DISCLAIMER}

This report was prepared as an account of work sponsored by an agency of the United States Government. Neither the United States Government nor any agency Thereof, nor any of their employees, makes any warranty, express or implied, or assumes any legal liability or responsibility for the accuracy, completeness, or usefulness of any information, apparatus, product, or process disclosed, or represents that its use would not infringe privately owned rights. Reference herein to any specific commercial product, process, or service by trade name, trademark, manufacturer, or otherwise does not necessarily constitute or imply its endorsement, recommendation, or favoring by the United States Government or any agency thereof. The views and opinions of authors expressed herein do not necessarily state or reflect those of the United States Government or any agency thereof. 


\section{DISCLAIMER}

Portions of this document may be illegible in electronic image products. Images are produced from the best available original document. 


\section{TRIP-I}

A TWO-DIMENSIONAL P-3 PROGRAM IN X-Y GEOMETRY

FOR THE IBM-704

E. Gelbard

J. Davis

J. Dorsey

H. Mitchell

J. Mandel

Contract AT-11-1-GEN-14

July 1960

Price $\$ .75$

Available from the Office of Technical Services, Department of Commerce, Washington 25, D. C。

NOTE

Thie document is an interim memnrandum prepared primarily for internal reference and does not represent a final expression of the opinion of Westinghouse. When this memorandum is distributed externally, it is with the express understanding that Westinghouse makes no representation as to completeness, accuracy, or usability of information contained therein. 


\section{SPECIAL EXTERNAL DISTRRIBUTION}

Director, Development Division; PNROO, AEC

Argonne National Laboratory, W: F: Miller Brookhaven National Laboratory, Jo Chernick Brookhaven National Laboratory, Mo Rose Case Institute of Technology, $R_{0} S$. Varga David Taylor Model Basin, H. Polachek Knolls Atomic Power Laboratory, R。 Ehrlich Los Alamos Scientific Laboratory, B. Carlson New York University; $R_{0}$ Richtmyer Oak Ridge National Laboratory, Vo. E. Anderson Oak Ridge National Laboratory; A. Householder University of California Radiation Laboratory, Livermore, S. Fermbach

\section{LEGAL NOTICE}

This report was prepared as an account of Government sponsored work. Neither the United States, nor the Commission, nor any person acting on behalf of these agencies:

A. Makes any warranty or representation; expressed or implied, with respect to the accuracy, completeness, or usefulness of the information contained in this report, or, that the use of any information, apparatius method, or process. disclosed in this report may not infringe privately owned rights; or

B. Assumes any liabilities with respect to the use of, or for damages resulting from the use of any information, apparatis, method, or process disclosed in this reportin

As used in the above, "person acting on behalf of these agencies" includes any employe or contractor of these agencies to the extent that such employe or contractor prepares, handles or distributes, or provides access to, any information pursuant to his employment or contract with the Commission. 
TABLE OF CONTENTS

$\underline{\text { Page }}$

I. INTRODUCTION

II. DERIVATION OF DIFFERENTIAL EQUATIONS

III. DERIVATION OF DIFFERENCE EQUATIONS : 6

IV. METHOD OF SOLUTION $\ldots 9$

V. CONVERGENCE CRITERIA 10

VI. DIFFERENCE EQUATIONS IN DETAIL . . . II

VII. METHOD OF SOLUTION FORMULATED IN MATRIX NOTATION 14

$\begin{array}{ll}\text { VIII. INPUT PREPARATION } & 15\end{array}$

$\begin{array}{ll}\text { IX。 EDITS } & 19\end{array}$

X. OPERATING INSTRUCTIONS 19 


\section{ABSTRACT}

TRIP-1 solves the one-group $P_{3}$ equations in $X Y$

geometry: It has been usêd to investigate two-dimensional

transport effects, and to study the feasibility of the method

of solution. The program is an extensively modified version

of $\mathrm{PDQ}-3(\operatorname{Ref} .3)$.

$\therefore$ 
TRIP-1

A TWO-DIMENSIONAL P-3 PROGRAM IN X-Y GEOMETRY

FOR THE IBM-704

E. Gelbard, J. Davis, J. Dorsey, H. Mitchell, J. Mandell

\section{INTRODUCTION}

The TRIP-1 program, written for the IBM-704, is designed to solve the $P_{3}$ equations in $X-Y$ geometry. Since two-dimensional transport effects are not yet well understood, TRIP-1 has been regarded, primarily, as an exploratory device rather than a production tool. Consequently all non-essential features have been excluded from the program, which treats only one-group cell problems. The cell is assumed to be rectangular, with regionwise constant cross sections. The source is isotropic and regionwise flat. Anisotropic scattering is dealt with rigorously (within the limitations of a $P_{3}$ approximation) and $P_{0}$ through $P_{3}$ scattering components are allowed.

In two dimensions, as in one, the $\mathrm{P}_{3}$ approximation has important limitations. For this reason, a cell problem should be examined closely before the TRIP-I solution is requested. It is often possible to assess the accuracy of $P_{3}$ in a given $X-Y$ problem through study of related one-dimensional problems. Further, it may be desirable in some cases to check selected features of the TRIP-l solution against a Monte Carlo calculation.

The derivation of TRIP-I finite difference equations is discussed in Section III, below. It will be seen that the TRIP-I and PDQ (Ref. I) difference equations are quite similar, and that the order of accuracy of the difference equations is the same in both programs. In general, however, the $\mathrm{P}_{3}$

\footnotetext{
The program requires 32,768 words of core storage. See Section $X$ for other requirements.
} 
scalar flux varies more sharply with position than does the $P_{1}$ This is particularly true in the neighborhood of interfaces If the fine detail of the $P_{3}$ solution 1 s not to be lost or distorted, it may be necessary to use more mesh points in a TRIP-1 problem than in the corresponding PDQ. Agaln, it is often possible to arrive at a satisfactory and economical mesh structure through a one-dimensional $P_{3}$ study. Since TRIP-1 aliows no more than 2500 interior mesh points, and since the minning time increases rapidly with the number of points, the mesh layout should be chosen with great care.

\section{DERIVATION OF DIFFERENTIAL DQUATIONS}

It is convenient to take as a point of departure the spherical har monics equations in Davioonilig: (Ref.2) compact notation:

$$
\begin{aligned}
(2 n-1) U \bar{U} \cdot \nabla_{r} & \psi_{n-1}-U^{2} \nabla_{u} \cdot \nabla_{r} \psi_{n-1}+\nabla_{u} \cdot \dot{\nabla}_{r} \psi_{n+1} \\
& +(2 n+1)\left(\Sigma_{T}-\Sigma_{s n}\right) \psi_{n}=(2 n+1) S_{n}
\end{aligned}
$$

Here $\Sigma_{s n}$ is the $n$ !th Legendro component of the scattering cross section. Equations (1) are identical with Davisonls; except for the addition of anisotropic source and scattering terms. In a $P_{3}$ approximation

$$
\begin{gathered}
\nabla_{u} \cdot \nabla_{r} \psi_{1}+\Sigma_{0} \psi_{0}={ }_{0} \\
\nabla_{u} \cdot \nabla_{r} \dot{\psi}_{2}+\bar{U} \cdot \nabla_{r} \psi_{0}+3 \Sigma_{1} \psi_{1}=0
\end{gathered}
$$




$$
\begin{aligned}
& \nabla_{u} \cdot \nabla_{r} \psi_{3}+3 U \cdot \nabla_{r} \psi_{I}-U^{2} \nabla_{u} \cdot \nabla_{r} \psi_{1}+5 \Sigma_{2} \psi_{2}=0 \text {, and } \\
& 5 \bar{U} \cdot \nabla_{r} \psi_{2}-U^{2} \nabla_{u} \cdot \nabla_{r} \psi_{2}+7 \Sigma_{3} \psi_{3}=0
\end{aligned}
$$

if the source is isotropic.

Upon elimination of the odd moments, one is led to two second-order equations:

$$
\begin{gathered}
-\nabla_{r} \cdot\left\{\frac{1}{3 \Sigma_{1}}\left[\nabla_{u}\left(\nabla_{u} \cdot \nabla_{r}\right) \psi_{2}+\nabla_{u}\left(\bar{U} \cdot \nabla_{r}\right) \psi_{0}\right]\right\}+\Sigma_{0} \psi_{0}=S_{0} \text {, and } \\
-\nabla_{r} \cdot\left\{\frac{1}{7 \Sigma_{3}}\left[5 \nabla_{u}\left(\bar{U} \cdot \nabla_{r}\right) \psi_{2}-\nabla_{u}\left(U^{2} \nabla_{u} \cdot \nabla_{r}\right) \psi_{2}\right]-\frac{1}{\Sigma_{1}}\left[\bar{U}\left(\nabla_{u} \cdot \nabla_{r}\right) \psi_{2}\right.\right. \\
\left.\left.+U\left(\bar{U} \cdot \nabla_{r}\right) \psi_{0}\right]\right\} \\
+5 \Sigma_{2} \psi_{2}=U^{2}\left(S_{0}-\Sigma_{0} \psi_{0}\right)
\end{gathered}
$$

In the above equations $\psi_{0}$ is simply the scalar flux, henceforth denoted by

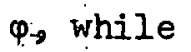

$$
\begin{aligned}
\psi_{2}=\frac{1}{5} \mathrm{U}^{2}\left[\psi_{20} \mathrm{P}_{2}^{0}(\mu)+\psi_{21} \cos \lambda \mathrm{P}_{2}^{1}(\mu)+\psi_{21}^{\prime} \sin \lambda \mathrm{P}_{2}^{1}(\mu)\right. \\
\left.+\psi_{22} \cos (2 \lambda) \mathrm{P}_{2}^{2}(\mu)+\psi_{22}^{\prime} \sin (2 \lambda) \mathrm{P}_{2}^{2}(\mu)\right]
\end{aligned}
$$

The angles $\lambda$ and $\theta=\cos ^{-1} \mu$ are, respectively, azimuthal and polar angles with respect to the $z$ axis, while the $P_{\ell}^{m}$ are associated Legendre polynomials. Introducing the components of $\overline{\mathrm{U}}$, one may write: 


$$
\begin{aligned}
\psi_{2} \equiv \frac{1}{5}\left[\frac{1}{2}\left(3 v_{z}^{2}-U^{2}\right) \psi_{20}\right. & +3 u_{x z} \psi_{21}+3 u_{y z} u_{21}+6 v_{x y} \psi_{22} \\
& \left.+3\left(u_{x}^{2}-u_{y}^{2}\right) \psi_{22}\right]
\end{aligned}
$$

If the vector flux is symmetric about the xy plane, then $\psi_{21}=\psi_{21}^{\prime}=0$. In terms of the new variables

$$
\begin{aligned}
& M \equiv-\frac{1}{10} \psi_{20}+\frac{3}{5} \psi_{22} \\
& P \equiv-\frac{1}{10} \psi_{20}-\frac{3}{5} \psi_{22} \text { and } \\
& B \equiv \frac{6}{5} \psi_{22}^{\prime},
\end{aligned}
$$

$\psi_{2}$ then takes the form

$$
\psi_{2} \equiv\left(U_{x}^{2}-U_{z}^{2}\right) M+\left(U_{y}^{2}-U_{z}^{2}\right) P+U_{\mathbf{x}} U_{y}^{B} B_{0}
$$

Substituting this explicit expression for $\psi_{2}$ into Eq. (6), one finds that

$$
-\dot{\nabla}_{r} \cdot\left\{\frac{1}{3 \Sigma_{1}}\left[\left(\frac{\partial m}{\partial x}+2 \frac{\partial M}{\partial x}+\frac{\partial B}{\partial y}\right) \hat{i}+\left(\frac{\partial \varphi}{\partial y}+2 \frac{\partial P}{\partial y}+\frac{\partial B}{\partial x}\right) \hat{j}\right]\right\}+\Sigma_{0} \varphi=s_{0}
$$

Similarly, definition (13) may be introduced into Bq. (7). It is easy to show that the resulting coefficients of $U_{x}^{2}, U_{y}^{2}, U_{z}^{2}$ and $U_{x} U_{y}$ must vanish. In consequence, four conditions are imposed on the moments, but only three are linearly independent: 


$$
\begin{aligned}
& -\nabla_{\mathbf{r}} \cdot\left\{\left[\frac{1}{7 \Sigma_{3}}\left(9 \frac{\partial M}{\partial x}-3 \frac{\partial B}{\partial y}\right)+\frac{1}{\Sigma_{1}}\left(2 \frac{\partial M}{\partial x}+\frac{\partial B}{\partial y}+\frac{\partial \varphi}{\partial x}\right)\right]\right\} \hat{i} \\
& \left.+\left[\frac{i}{7 \Sigma_{3}}\left(4 \frac{\partial B}{\partial x}+5 \frac{\partial M}{\partial y}-2 \frac{\partial P}{\partial y}\right)\right] \hat{j}\right\} \\
& +5 \Sigma_{2} M-s_{0}-\Sigma_{0} \\
& -\nabla_{r}\left\{\left\{\left[\frac{1}{7 \Sigma_{3}}\left(9 \frac{\partial P}{\partial y}-3 \frac{\partial B}{\partial x}\right)+\frac{1}{\Sigma_{1}}\left(2 \frac{\partial P}{\partial y}+\frac{\partial B}{\partial x}+\frac{\partial \varphi}{\partial y}\right)\right] \quad \hat{j}\right.\right. \\
& \left.+\left[\frac{1}{7 \Sigma_{3}}\left(4 \frac{\partial B}{\partial y}+5 \frac{\partial P}{\partial x}-2 \frac{\partial M}{\partial x}\right)\right] \quad \begin{array}{l}
\hat{i} \\
\vdots
\end{array}\right\} \\
& +5 \Sigma_{2} P=S_{0}-\Sigma_{0} \varphi \quad \text { and } \\
& -\nabla_{r}:\left\{\left[\frac{1}{7 \Sigma_{3}}\left(8 \frac{\partial B}{\partial x}+10 \frac{\partial M}{\partial y}-4 \frac{\partial P}{\partial y}\right)+\frac{1}{\Sigma_{1}}\left(\frac{\partial B}{\partial x}+2 \frac{\partial P}{\partial y}+\frac{\partial Q}{\partial y}\right)\right] \hat{i}\right. \\
& +\left[\frac{1}{7 \Sigma_{3}}\left(8 \frac{\partial B}{\partial y}+10 \frac{\partial P}{\partial x}-4 \frac{\partial M}{\partial x}\right)+\frac{1}{\Sigma_{1}}\left(\frac{\partial B}{\partial y}+2 \frac{\partial M}{\partial x}+\frac{\partial \varphi}{\partial x}\right)\right]\left\{\begin{array}{l}
\hat{j} \\
j
\end{array}\right. \\
& +5 \Sigma_{2} B=0
\end{aligned}
$$

The functions $\varphi, M, P$ and $B$ are, then, determined by Eqs. (14) through (17), supplemented by approximate boundary conditions. From the definitions of these functions it follows that $\varphi ; M$ and $P$ are symetric about the cell boundaries, while $B$ is antisymetric. ${ }^{t}$ Continuity conditions at

* It is assumed here that the boundaries are parallel to the coordinate axes. 
interfaces will be stated in section III, below.

It has been verified that Egs. (14) through (17) are lnvartant under $45^{\circ}$ and $90^{\circ}$ rotation, and that they pass to the correct one-dimenolonel jimit. From (13), it follows that

$$
\begin{aligned}
& F_{2 x} \equiv \int \frac{\left(3 \Omega_{x}^{2}-1\right)}{2} F(\hat{\Omega} ; \bar{x}) d \hat{\Omega}=M \text { and } \\
& F_{2 y} \equiv \int \frac{\left(3 \Omega_{y}^{2}-1\right)}{2} F(\hat{\Omega}, \bar{r}) d \hat{\Omega} P
\end{aligned}
$$

Therefore, in one-dimensional $\mathrm{x}$ and $\mathrm{y}$ geometries, $\mathrm{M}$ and $\mathrm{P}$, respectivel, $\mathrm{y}$, should play the role of $F_{2}$. Examination of Eqs. (14) through (17) will show that this iș, indeed, the case.

\section{DERIVATION OF DIFFERENCE EQUATIONS}

The two-dimensional $P_{3}$ equations may be cast into finte difference form, and then solved by standard iteration procedures. In the foliowing discussion it will be assumed that all interfaces lie on mesh Innes." Nine neighboring pesh points are shown in Fig. Io Difference equations at point 0 wili be derived by integration; as in $\mathrm{PDQ}$. In order to illustrate this procedure,

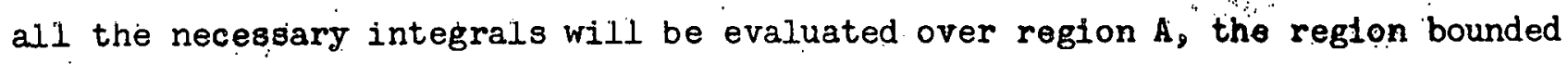
by lines $(0,9),(9,10),(10,11)$ and $(11,0)$. First it is convenient to abbreviate the $P_{3}$ equations which may be written in the simple form:

$$
\nabla_{r} \cdot \vec{V}_{I}+F_{I}=0, \cdots I \leq I \leq 4 .
$$


MESH RECTANGLE SURROUNDING ZEROTH MESH POINT

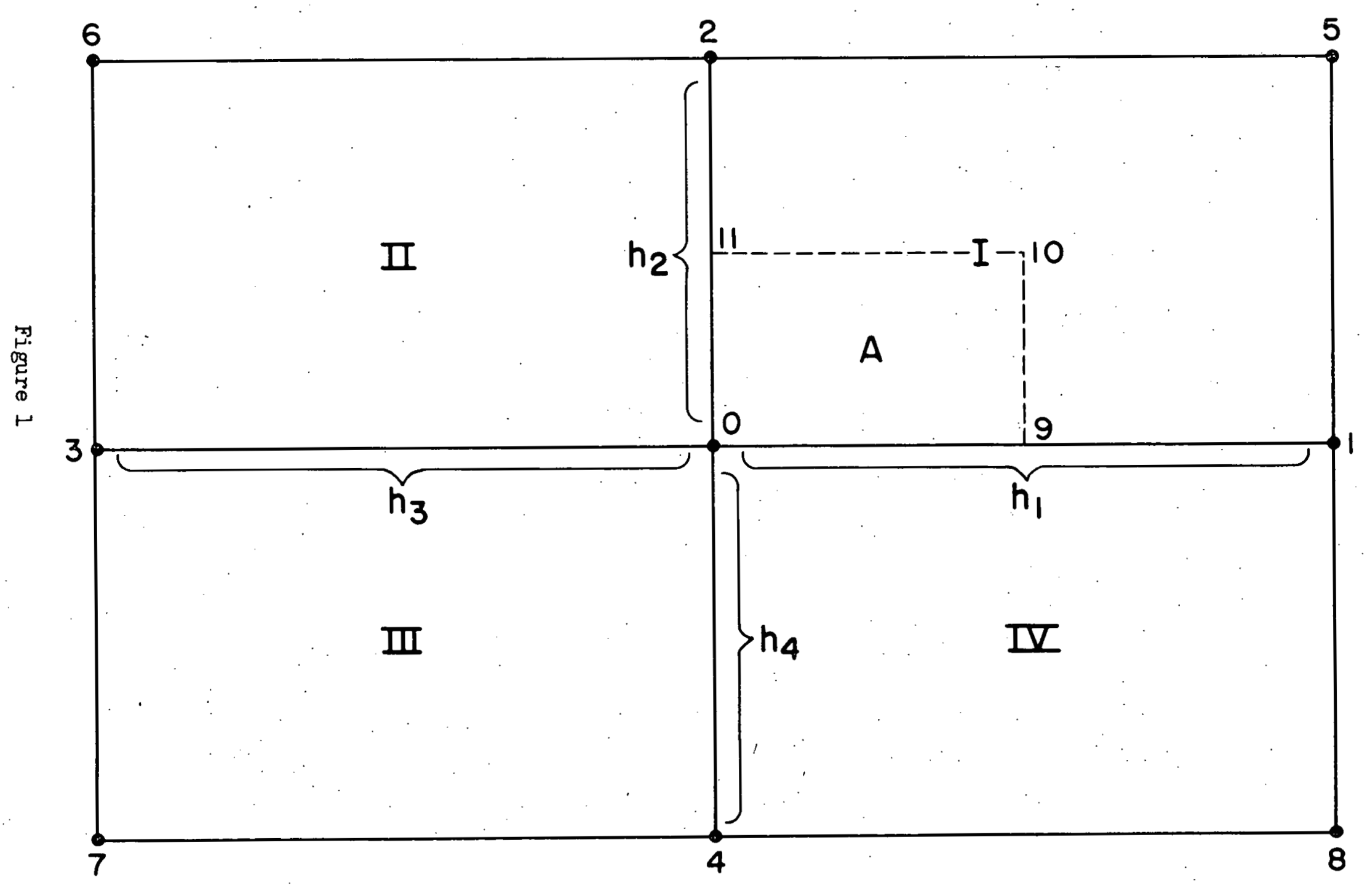


As in PDQ,

$$
\int_{A} \nabla_{r} \cdot \bar{V}_{I} d A+\int_{A} F_{I} d A \approx \int_{C} V_{I n} d S+\frac{1}{4} h_{I} h_{2} F_{I O} ?
$$

where $V_{\text {In }}$ is the normal component of $\bar{V}_{I^{\prime}}$ Ultimately, contributions from the four quadrants surrounding point 0 will be added. Now, it can be shown, through a "pilibox" argument, that $V_{\text {In }}$ is continuous across interfaces. Consequently, the line integrals along segments $(0,9)$ and $(0,11)$ will be cancelled by contributions from other quadrants. Only integrals along $(9,10)$ and $(10,11)$ need be computed. These integrals consist of sums of terms, of the following types:

$$
\begin{gathered}
I_{1}=\int_{(9)} \frac{\partial}{\partial x} G(x, y) d s, \\
I_{2}=\int_{(9)}^{(10)} \frac{\partial}{\partial y} G(x, y) d s,
\end{gathered}
$$

$$
I_{3}=\int_{(10)}^{(11)} \frac{\partial}{\partial y}(t), \quad \therefore
$$

$$
I_{4}=\int_{(10)} \frac{\partial}{\partial x} G(x, y) d s,
$$

where the symbol $G(x, y)$ may deșignate any one of the functions $\varphi, M_{0} P$ or $B_{0}$ Integrals of type 20 and 22 occur in the derivation of PDQ difference equations. They will be approximated as in PDQ:

$$
I_{1} \approx \frac{h_{2}}{2}\left[\frac{G_{1}-G_{0}}{h_{1}}\right],
$$




$$
I_{3} \approx \frac{h_{1}}{2}\left[\frac{G_{2}-G_{0}}{h_{2}}\right]
$$

To the same order

$$
\begin{aligned}
& I_{2} \approx \frac{I}{2}\left[\frac{G_{2}+G_{5}-\left(G_{0}+G_{1}\right)}{2}\right], \text { and } \\
& I_{4}=\frac{1}{2}\left[\frac{G_{2}+G_{5}-\left(G_{0}+G_{2}\right)}{2}\right],
\end{aligned}
$$

The above approximations define the TRIP-1 difference equations. Boundary conditions may be imposed without difficulty. The cell boundaries are located halfway between mesh rows or mesh columns. Suppose, for example, that a cell boundary passes between points 0 and 1 (Fig. 1). Then, for symmetric G's, $G_{5}=G_{2}, G_{1}=G_{0}, G_{8}=G_{4}:$. If $G$ is to be antisymmetric, $G_{5}=-G_{2}, G_{1}=-G_{0}$, $G_{8}=-G_{4}$ ?

The exact form of the difference equations is exhibited in fill detail in Section VI.

\section{METHOD OF SOLUTION}

Equations (14) through (17), in difference form; are solved by a combination of inner and outer iterative processes. Equation (14) is used to compute $\varphi$, with terms in $M, P$ and $B$ treated as constituents of the source: Similarly, Eqs. (15), (16) and (17) are solved for $M, P$ and $B$, respectively. A regionwise flat guess for $\Phi$ is entered as input; initial values of M, $\mathrm{P}$ and $\mathrm{B}$ are taken to be zero. Equation (14) is then solved by simultaneous line overrelaxation, as in $\mathrm{PDQ}-3$ (Ref. 3) using $\mathrm{PDQ}-3$ routines. It should be 
noted that Eq (14), with $\mathrm{M}_{9} \mathrm{P}$ and $\mathrm{B}$ set equal to zero, is simply the $\mathrm{P}_{1}$ diffusion equation:

Having solved Eq (14), the newly computed $\varphi$ is used to calculate the source in Eq. (15), (with $P$ and $B$ still zero); Eq. (15) is solved nexto Subsequently, Eq. (16) and (17) are solved (in that order); whereupon the outer: cycle is repeated.

Throughout the outer cycles, all source terms are computed from the iatest Iterates of the moments. All equations are solved by simultaneous line overrelaxation, with optimum $\omega^{\text {? }}$ s computed for each equation. These $\omega^{8}$ s are determined during the course of the first outer iteration. Convergence of the outer cycle is not accelerated, but the unaccelerated convergence rate is generally fast.

\section{V:. CONYERGENGE CRITERIA:}

Separate convergence criteria are used to terminate the inner and outer fterative processes. Inner iteration is continued, in each pquation, untı?

$$
\left|\frac{G_{I}^{(n+1)}-G_{I}^{(n)}}{G_{I}^{(n+I)}}\right|<\varepsilon\left(2-w_{I}\right)
$$

at all mesh points. Here $G_{I}^{(n)}$ and $a_{I}^{(n+1)}$ denote successive inner iterates of tho Independent variable th the foth equation, while wi th the computed ovap refaxation factor. It will be seen that a single $\varepsilon$ is opeciffod for all fqur equations However, the factor $2-\omega_{I}$ automatically refines the confergenoe criterion when the convergence rate is low. As in $\mathrm{PDQ}-3$, this device is uned to prérent fálse convergence. 
The outer cycle is terminated when

$$
\frac{\varphi^{(N+1)}-\varphi^{(N)}}{\varphi^{(N+1)}} \leq \eta
$$

In $\mathrm{Eq}_{0}(28) \varphi^{(\mathrm{N}+\mathrm{I})}$ and $\varphi^{(\mathrm{N})}$ denote successive outer iterates of the scalar flux. No attempt is made, here, to adjust the convergence criterion to the convergence rate. This precaution does not seem to be necessary in view of the rapid cyclo. Most TRIP- 1 problems have been run, at Bettis, with $\eta=001, \varepsilon=001$ It should be noted that the TRIP-I $\varepsilon$ is, generally, coarser than the inner cycle criterion required in a one-energy $P_{1}$ problem. This is permissible since each TRIP-I equation 1 s entered repeatedly during successive outer cycles.

VI. DIFFERENCE EQUATIONS IN DETAIL

The TRIP-1 difference equations are exhibited belaw.

$-\left[\sum_{n=1}^{4} \alpha_{n}+\frac{3}{2} x\right] \frac{\Phi_{0}}{2}+\frac{1}{2} \sum_{n=1}^{4} \alpha_{n} \phi_{n}-\left(\alpha_{1}+\alpha_{3}\right) M_{0}+\alpha_{1} M_{1}+\alpha_{3} M_{3}-\left(\alpha_{2}+\alpha_{4}\right) P_{0}$

$$
+\alpha_{2} p_{2}+\alpha_{4} P_{4}+\frac{\delta}{2} B_{0}+\frac{1}{2} \sum_{n=5}^{8}(-1)^{n+1} \frac{B_{n}}{\delta_{n-4}}=-\frac{3}{4} \xi
$$

$-\left[\alpha_{1}+\alpha_{3}+\frac{9}{14}\left(\omega_{1}+\omega_{3}\right)+\frac{5}{14}\left(\omega_{2}+\omega_{4}\right)+\frac{5}{4} Y\right] M_{0}+\left(\alpha_{1}+\frac{9}{14} \omega_{1}\right) M_{1}$

$+\left(\frac{5}{14} \omega_{2}\right) M_{2}+\left(\alpha_{3}+\frac{9}{14} \omega_{3}\right) M_{3}+\left(\frac{5}{14} \omega_{4}\right) M_{4}-\left(\alpha_{1}+\alpha_{3}+\frac{X}{2}\right) \frac{\Phi_{0}}{2}+\left(\frac{\alpha_{1}}{2}\right) \varphi_{1}$

Subscripts on $\Phi, M, P$ and $B$ denote mesh points, as in Fig. 10 
$+\left(\frac{\omega_{3}}{2}\right) \varphi_{3}+\frac{\left(\omega_{2}+\omega_{4}\right)}{7} P_{0}-\left(\frac{\omega_{2}}{7}\right) \cdot P_{2}-\left(\frac{\omega_{4}}{7}\right) P_{4}+\left(\frac{\bar{\nu}}{7}\right) \frac{B_{0}}{4}$

$+\frac{1}{4} \sum_{\substack{n=1 \\ 1 \leq(n-1) \leq 4}}^{4} B_{n} C_{n, n-1}+\frac{1}{4} \sum_{n=5}^{8}(-1)^{n+1} B_{n}{ }^{2}=-\frac{\xi}{4}$

$-\left[\alpha_{2}+\alpha_{4}+\frac{5}{14}\left(\omega_{1}+\omega_{3}\right)+\frac{9}{14}\left(\omega_{2}+\omega_{4}\right)+\frac{5}{4} Y\right] P_{0}+\left(\frac{5}{14} \omega_{1}\right) P_{1}$

$+\left(\omega_{2}+\frac{\rho}{14} \omega_{2}\right) r_{2}+\left(\frac{5}{14} \omega_{3}\right) p_{3}+\left(\alpha_{4}+\frac{9}{14} \omega_{4}\right) p_{4}-\left(\alpha_{2}+\alpha_{4}+\frac{\dot{x}}{2}\right) \frac{\varphi_{0}}{2}$

$+\left(\frac{\alpha_{2}}{2}\right) \Phi_{2}^{\prime}+\left(\frac{\alpha_{4}}{2}\right) \varphi_{4}+\frac{\left(\omega_{1}+\omega_{3}\right)}{7} M_{0}-\left(\frac{\omega_{1}}{7}\right) M_{1}-\left(\frac{\omega_{3}}{7}\right) M_{3}+\left(\bar{\sigma}+\frac{\bar{\mu}}{7}\right) \frac{0}{4}$

$-\frac{1}{4} \sum_{1 \leq n-1 \leq 4}^{4} B_{n} C_{n, n-1}+\frac{1}{4} \sum_{n=5}^{8}(-1)^{n+1} B_{n} A_{n}=-\frac{8}{4} ;$

$-\left[\sum_{n=1}^{4}\left(\alpha_{n}+\frac{8}{7} \omega_{n}\right)+\frac{5}{2} Y\right] B_{0}+\sum_{n=1}^{l}\left(\alpha_{n}+\frac{8}{7} \omega_{n}\right) B_{n}+(\bar{\delta}) \varphi_{0}+\sum_{n=5}^{8}(-1)^{n+1} \frac{\varphi_{n}}{\delta_{n-4}}$

$+\left(\delta+\frac{3}{7} \vec{\mu}\right) P_{0}+\sum_{1 \leq n=1}^{4} c_{n, n-1} P_{n}+\sum_{n=5}^{8}(-1)^{n+1} b_{n} P_{n}+\left(\delta+\frac{3}{7} \vec{\mu}\right) M_{0}$

$-\sum_{\substack{n=1 \\ n=1}}^{4} c_{n, n-1} \mathbb{M}_{n}+\sum_{n=5}^{8}(-1)^{n+1} b_{n}=0$

where $\varphi, M_{3} P$ and $B$ are the variables, $\chi \chi \equiv \Sigma_{0}, \delta \equiv \Sigma_{1}, \rho \equiv \Sigma_{2}, \mu \equiv \Sigma_{3}$

and $S \equiv S_{0}$. 
Equations 29 through 32 are derived from 14 through 17 , respectively.

Coefficients in the difference equations are defined through the following relations:

$$
\begin{aligned}
& \delta=-\frac{1}{\delta_{1}}+\frac{1}{\delta_{2}}-\frac{1}{\delta_{3}}+\frac{1}{\delta_{4}} \\
& \stackrel{m}{\mu}=-\frac{1}{\mu_{1}}+\frac{1}{\mu_{2}}-\frac{1}{\mu_{3}}+\frac{1}{\mu_{4}} \text {. } \\
& \alpha_{1}=\frac{1}{h_{1}}\left(\frac{h_{2}}{\delta_{1}}+\frac{h_{4}}{\delta_{4}}\right) \\
& \omega_{1}=\frac{1}{h_{1}}\left(\frac{h_{2}}{\mu_{1}}+\frac{h_{4}}{\mu_{4}}\right) \text {. } \\
& \alpha_{2}=\frac{1}{h_{2}}\left(\frac{h_{3}}{\delta_{2}}+\frac{h_{1}}{\delta_{1}}\right) \\
& \omega_{2}=\frac{1}{h_{2}}\left|\frac{h_{3}}{\mu_{2}}+\frac{h_{1}}{\mu_{1}}\right| \text {. } \\
& \alpha_{3}=\frac{1}{h_{3}}\left(\frac{h_{4}}{\delta_{3}}+\frac{h_{2}}{\delta_{2}}\right) \ldots \\
& \omega_{3}=\frac{1}{h_{3}}\left|\frac{h_{4}}{\mu_{3}}+\frac{h_{2}}{\mu_{2}}\right| \\
& \alpha_{4}=\frac{1}{h_{4}}\left(\frac{h_{1}}{\delta_{4}}+\frac{h_{3}}{\delta_{3}}\right) \\
& \omega_{4}=\frac{1}{h_{4}}\left(\frac{h_{1}}{\mu_{4}}+\frac{h_{3}}{\mu_{3}}\right) \text {. } \\
& a_{n}=\frac{1}{\delta_{n-4}}+\frac{1}{7 \mu_{n-4}} \\
& b_{n}=\frac{1}{8_{n-4}}+\frac{3}{7 \mu_{n-4}} \text {. } \\
& c_{14}=\frac{1}{\delta_{1}}+\frac{1}{\mu_{1}}+\frac{1}{\delta_{4}}-\frac{1}{\mu_{4}} \\
& c_{2 I}=-\frac{1}{\delta_{2}}+\frac{1}{\mu_{2}}+\frac{1}{\delta_{1}}-\frac{1}{\mu_{1}} \\
& \sigma_{32}=-\frac{1}{\delta_{3}}+\frac{1}{\mu_{3}}+\frac{1}{\delta_{2}}-\frac{1}{\mu_{2}} \\
& s_{43}=-\frac{1}{\delta_{4}}+\frac{1}{\mu_{4}}+\frac{1}{\delta_{3}}-\frac{1}{\mu_{3}} \\
& Y=\rho_{1} h_{1} h_{2}+\rho_{2} h_{2} h_{3}+\rho_{3} h_{3} h_{4}+\rho_{4} h_{4} h_{1} \\
& x=x_{1} h_{1} h_{2}+x_{2} h_{2} h_{3}+x_{3} h_{3} h_{4}+x_{4} h_{4} h_{1} \\
& \xi=s_{1} h_{1} h_{2}+s_{2} h_{2} h_{3}+s_{3} h_{3} h_{4}+\delta_{4} h_{4} h_{1}
\end{aligned}
$$

$-13=$ 
Boundary conditions have already beer discussed in Section III.

VII. METHOD OF SOLUTION FORMULATED IN MATRIX NOTATION.

To simplify notation, it is convenient to introduce the vectors $\Phi_{1} \approx \Phi_{9} \Phi_{2} \approx M_{9} \Phi_{3} \approx P_{9} \Phi_{4} \approx B_{9}$ vectors whose components are ordered as In PDQ-3. The TRIP-1 difference equations can then be cast into matrix form, as follows:

$$
\begin{aligned}
& A_{11} \Phi_{1}=A_{12} \Phi_{2}+A_{13} \Phi_{3}+A_{14} \Phi_{4}+\Psi_{1} \\
& A_{22} \Phi_{2}=A_{21} \Phi_{1}+A_{23} \Phi_{3}+A_{24} \Phi_{4}+\psi_{2} \\
& A_{33} \Phi_{3}=A_{31} \Phi_{1}+A_{32} \Phi_{2}+A_{34} \Phi_{4}+\psi_{3} \\
& A_{44} \Phi_{4}=A_{41} \Phi_{1}+A_{42} \Phi_{2}+A_{43} \Phi_{3}
\end{aligned}
$$

These matfix equations are solved by a Gauss-Seidel 1terative process; defined below:

$$
\begin{aligned}
& A_{11} \Phi_{1}^{(N+1)}=A_{12} \Phi_{2}^{(N)}+A_{13} \Phi_{3}^{(N)}+A_{14} \Phi_{4}^{(N)}+\psi_{1} \\
& A_{22} \Phi_{2}^{(N+I)}=A_{21} \Phi{ }_{1}^{(N+1)}+A_{23} \Phi{ }_{3}^{(N)}+A_{24} \Phi{ }_{4}^{(N)}+\psi_{2} \\
& A_{33}{ }_{3}^{(N+1)}=A_{31}^{\Phi}{ }_{1}^{(N+1)}+A_{32} \Phi_{2}^{(N+1)}+A_{34} \Phi_{4}^{(N)}+\psi_{3} \text {, } \\
& A_{44} \Phi_{4}^{(N+1)}=A_{41} \Phi_{1}^{(N+1)}+A_{42} \Phi_{2}^{(N+1)}+A_{43} \Phi_{3}^{(N+1)}:
\end{aligned}
$$

Equations 37 through 40 may be condensed further:

$$
\begin{aligned}
A_{I I}{ }_{I}^{(N+1)} & =\Psi \\
& =14
\end{aligned}
$$


where $\Psi(\mathrm{N}+\mathrm{I})$ is known. The function $\Phi_{I}^{(N+1)}$ is computed by a simultaneous line overrelaxation process, as in PDQ-3.

\section{INPUT PREPARATION}

The routine which processes the input is a slightly modified version of that used in PDQ-3.

\section{Card Input}

All of the input to this program is from punched cards.

Title Card. A title card must precede the input deck of each problem. Columns 1-8 must contain an eight-digit numeric identification. Columns 65-67 must be blank. Columns 68-72 must contain TRIPI.

Input Deck. All input numbers are expressed in the fixed-point form. The card format is such that columns $1-7$ and 11 are blank, columns 8-10 contain "DEC", and columns $12-15$ contain the card number, followed by a comma in column 16. The input parameters begin in column 17 and may extend through column 72. Successive numbers are separated by commas, but no comma is allowed following the last number on a card. The first blank column indicates the end of the information on that card. The numbers may be preceded by signs, but only minus signs are necessary。

The number zero need not have a decimal point. The nonzero numbers on card 1002, those contained in the 2000 and 3000 series, and the mesh intervals in the 4000 and 5000 series must contain decimal points. The nonzero numbers on cards 1001 and 1003, the row and column numbers in the 
4000 and 5000 series, and the nonzero numbers in the 6000,7000 ; and 8000 series mast not contain decimal points A detailed description of each card follows.

\section{Card Number}

1001

1002

2101

2102

$21 \underline{n}$

Description

$K, n, s s, t g_{,}, b_{g}, c_{g} d$

$K$ must be one and $a=b=c=d=$ one $n$ is the largest composition number for which data is provided in the 2000 series ( $1 \leq n \leq 35$ )。 Data must be provided for ali compositions $c=1,2,00, n$ 。

88 is the last column in the mesh ( $3 \leq \mathrm{ss} \leq 87)$. Column ss. is half an interval beyond the symmetry boundary.

tt is the last row in the mesh $[3 \leq t t \leq 174$, ss $\leq t t$, $($ ss -1$)(t t-1) \leq 2500]$. Row $t t$ is half an interval beyond a symmetry boundary.

$\mathrm{f}_{9} \eta_{g} \varepsilon, \mathrm{g}_{g} \mathrm{~h}, \ell_{9}, \mathrm{~m}, \mathrm{n}$

$f, g, h, l, m$, and $n$ must always be zero.

$\eta$ is the parameter used for stopping the problem with the criterion being

$$
\frac{\left|\varphi_{i}^{(N+1)}-\varphi_{i}^{(N)}\right|}{\left|\varphi_{i}^{(N+1)}\right|} \leq n
$$

where $\dot{\varphi}_{i}^{(N)}$ is the i-th component of the flux, $\varphi$, after iteration $N$.

$E$ is lie paraneter used for stopping tho i.toration in oach of the four matrix equations. The criterion is

$$
\left|\frac{\Phi_{I I}^{(n+1)}-\Phi_{I !}^{(n)}}{\Phi_{I 1}^{(n+1)}}\right| \leq \varepsilon\left(z-\omega_{I}\right)
$$

where $\omega_{I}$ is the overrelaxation factor computed for matrix equation $I, I_{n}$ is the iteration index, and $\Phi_{I j}$ is the $i-t h$ component of $\Psi$ :

$$
\Sigma_{1}, \Sigma_{a}, \Sigma_{3}, \Sigma_{2}, S_{0}
$$

These are the composition-variable parameters for compositions 1 through $n_{0} \Sigma_{\eta}$ and $\Sigma_{3}$ must always be greater than zero and all the rest must be nonnegative: In the above notation, $\Sigma_{1}=$ $\Sigma_{a}+\Sigma_{S O}-\Sigma_{\text {Si }}$, for $1=1,2,3$ 。 
$\varphi$

$\varphi$ is a constant input flux approximation. This card is optional. If this card is present, there may be no 3000 series. If this card is absent, there must be a 3000 series.

3001

3002<smiles>C1=CCCCC1</smiles>

$30 \underline{n}$

4001 4002

5001

5002

6001

6002

$\varphi$

$\varphi$ is a flux approximation for compositions 1 through $n$. This series is optional. See card 3000 .

$\mathrm{H}_{9} \mathrm{~s}_{1}, \mathrm{H}, \mathrm{s}_{2}, \therefore \circ$

This is the sequence of mesh intervals in the $x$ coordinate direction. The first value is the mesh interval between columns $O$ and $s_{1}$, the second is the interval between columns $s_{1}$ and $s_{2}$ s and so forth. No set $\mathrm{H}, \mathrm{s}$, may overlap two cards, the sequence $s_{\text {i }}$ must be strictly increasing, and the last value must equal ss on card 1001 .

$H, t_{1}, H, t_{2}, \ldots$

This is the sequence of mesh intervals in the $\mathrm{y}$ coordinate direction. The first value is the mesh interval between rows 0 and $t_{1}$, the second is the interval between columns $t_{1}$ and $t_{2}$, and so forth. No set $H_{2} t_{i}$ may overlap two cards, the sequence ${ }^{2} t_{i}$ must be strictly increasing, and the last value must equal tt on card 1001 .

$c_{9}, s_{1}, s_{2}, t_{1}, t_{2}, \ldots$

The material composition described by successively laying rectangular blocks of specified composition over the mesh. Any block of composition may be laid over all or part of other blocks specified previously. For each mesh rectangle, the last speciom fication which includes this rectangle determines its composition. It is not necessary that every composition $c, l \leq c \leq n$, appear in the mesh.

$s_{1}$ s. $s_{2}, t_{1}$, and $t_{2}$ are the left-hand, right-hand, upper, and lower boundaries, respectively, of composition number $c$, where $0 \leq s_{1}<s_{2} \leq s s$ and $0 \leq t_{1}<t_{2} \leq t t_{\text {。 }}$

No specification of a composition block may overlap two cards. 
Bg so so $s_{i} t_{2} \circ \circ$

The integrals of the flux and the absorption are automatically calculated for each composition. If there are any additional rectangular regions over which the flux and absorption are to be integrated: they are specified in this series by five-word sets. The first word of a set, $g$, is one when designating the flux and is two when designating the absorption. "The next four words describe the outer boundaries of the rectangle over which the integration is desired. If a rectangle includes a symmetry boundary, the region outside this boundary is not considered.

$s_{1}, s_{2}, t_{1}, t_{2}$ are as defined in the description of the 6000 series.

The total number of rectangles spectifed in this series must not exceed 100 and the sequence of values chosen as g mat. be nondecreasing. No specification of a rectangle may overlap two cards. If there are no additional integrations to be requested in this series, a card numbered 7001 must be provided th no comma following the card number.

8001

$\pm g_{9} s_{1}, s_{2}, t_{1}, t_{2}, \ldots$

Pointwise editing is done only in those equations and orer those rectangles specified by five-word sets. The first word of a set is the equation number, $g_{8}$ and the next four words, $s_{1}, \theta_{2} ; t_{1}$, $t_{2}$. are as defined in the 6000 series except that here $I^{2} \leq s_{1}<s_{2} \leq s s-l_{g} 1 \leq t_{j}<t_{2} \leq t t-I_{\text {o }}$ and $1 \leq|g| \leq 40$. If $g$ is positive, the editing is done in fixedopoint with three digits to the right of the decimal point and up to four digits to the left. If $g$ is negative, the editing is done in floating-point with a two-digit exponent and $a$ four-digit mantissa.

The total number of rectangle specifications may not excoed 100. The sequence of values of $\mid$ g $\mid$. must be nondecreasing. No rectangle specification may overlap two cards. If there is no edit control inforiation, a oard 8001 nuat be provided with no comma following the card number。

This card signifies the end of the input. 
IX。 EDITS

All edits are accomplished by use of the $P D Q-3$ edit routines. At the end of the problem, the program provides the following edits:

(1) Input Edit

(2) Composition-Integrated Area: $\sum_{\mathrm{R}^{\prime}} \mathrm{h}_{\mathrm{s}} \mathrm{h}_{\mathrm{t}}$

(3) Composition-Integrated Scalar Flux, $\varphi$ :

$$
\sum_{R} h_{s} h_{t} \frac{\left\{\varphi_{s-1, t-1}+\varphi_{s-1, t}+\varphi_{s, t-1}+\varphi_{s, t}\right\}}{4}
$$

(4) Composition Averaged Flux:

$$
\bar{\varphi}_{\mathrm{R}} \equiv(3) \div(2)
$$

(5) Composition-Integrated Absorption: $\Sigma_{\mathrm{O}} \bar{\varphi}_{\mathrm{R}}$

(6) Composition-Averaged Absorption: (5) $\div$ (2),

where in the above expressions $R$ is the region of interest, $h_{s}$ is the interval between mesh columns $s-l$ and $s$, and $h_{t}$ is the interval between mesh-rows $t-l$ and t.

(7) Picture indicating mesh lines and compositions.

(8) Pointwise Edit ooneioting of $\Phi_{1}, \Phi_{2}, \Phi_{3}$, and $\Phi_{4}$ 。

X. OPERATING INSTRUCTIONS

Card Reader: Use the 72-72 card reader board.

On-Line Printer: Use the SHARE 2 or the GLOUT 2 printer board and paper which has space enough for at least 72 columns. 
Card Punch: "Standard SHARE Board:

Off-Iine Printer: Use 120 column paper and set the carriage control switch to PROGRAM.

Tapes: Use 9 tape units designated as 1-9 with the instruction tape designated as tape 1 .

Sense Switches: All switches are normally set to UP, If No: 2 is down this setting signifies a restart.

If 3 is down, this setting signt fies a forced edit.

Starting a Problem: See that the necessary tapes are mounted, that tape 1 is rewound, and that sense switches 2 and 3 are properly set. Then depress the CLEAR and LOAD TAPE ikeys.

Restart Procedure: To restart a problem at the last restart point, see that the original tape reels are remounted and set at the same logical settings as set initially. Then rewind tape 1, depress sense switch 2, CLEAR and LOAD TAPE。

Ramoving a Problem: To remove a problem from the computer, rewind, remove, and label tape 2-9:

Forced Edit: To force an edit at the end of the current iteration, depress sense switch 30 This will cause an edit to be written on tape 9. When continued, put on a new tape 9. (DO NOT depress sense switch 3 until the results of Eq. (1) are printèdo)

Tape output: The only output tape for off-line printing is tape 9.

Card Qutput: Each tape check sum or redundancy tape test error causes a card: to be punched. Rows $9 R$ and $8 R$ contein the check sum from tape and the computed check sum, respectively. If both $9 R$ and $8 R$ are zero, the error 1s due lu lis redundancy tape test. Row $2 R$ contains the record number and file number in the address and decrement, respectively. Row OR contains the RTB or WTB instruction.

Instruction Tape Preparation: To write an instruction tape, ready a blank tape 1 , ready the TRIP-I deck In the card reader, CLEAR, and LOAD CARDS. A stop at 343 indicates that the proper instruction tape consisting of 30 records has been written.

\section{Program Stops:}

00006: Error loading binary tape loader from tape 1。 Push LOAD TAPE to try again.

00077: Error loading a record of instructions from tape 1. Push START to troy again.

77777: The reason for this stop is printed on-line. 


\section{$\underline{\text { References }}$}

1. G. G. Bilodeau, et al, "PDQ $-\infty$ An IBM-704. Code to Solve the Two-Dimensional Few-Group Neutron-Diffusion Equations;" WAPD-TM-70, August 1957.

2. B. Davison, Neutron Transport Theory, Oxford (1957), p. 160.

3. W. R. Cadwell, "PDQ-3 - A Program for the Solution of the Neutron-Diffusion Equations in two Dimensions on the IBM-704," WAPD-TM-179, March 1960. 